\title{
Frugivores bias seed-adult tree associations through nonrandom seed dispersal: a phylogenetic approach
}

\author{
Onja H. RaZafindratsima ${ }^{1,2,3}$ and Amy E. Dunham ${ }^{1,2}$ \\ ${ }^{1}$ Department of Biosciences, Rice University, 6100 Main Street, Houston, Texas 77005 USA \\ ${ }^{2}$ BP 33, Centre ValBio, Ranomafana, Ifanadiana 312, Madagascar
}

\begin{abstract}
Frugivores are the main seed dispersers in many ecosystems, such that behaviorally driven, nonrandom patterns of seed dispersal are a common process; but patterns are poorly understood. Characterizing these patterns may be essential for understanding spatial organization of fruiting trees and drivers of seed-dispersal limitation in biodiverse forests. To address this, we studied resulting spatial associations between dispersed seeds and adult tree neighbors in a diverse rainforest in Madagascar, using a temporal and phylogenetic approach. Data show that by using fruiting trees as seed-dispersal foci, frugivores bias seed dispersal under conspecific adults and under heterospecific trees that share dispersers and fruiting time with the dispersed species. Frugivore-mediated seed dispersal also resulted in nonrandom phylogenetic associations of dispersed seeds with their nearest adult neighbors, in nine out of the 16 months of our study. However, these nonrandom phylogenetic associations fluctuated unpredictably over time, ranging from clustered to overdispersed. The spatial and phylogenetic template of seed dispersal did not translate to similar patterns of association in adult tree neighborhoods, suggesting the importance of post-dispersal processes in structuring plant communities. Results suggest that frugivoremediated seed dispersal is important for structuring early stages of plant-plant associations, setting the template for post-dispersal processes that influence ultimate patterns of plant recruitment. Importantly, if biased patterns of dispersal are common in other systems, frugivores may promote tree coexistence in biodiverse forests by limiting the frequency and diversity of heterospecific interactions of seeds they disperse.
\end{abstract}

Key words: contagious dispersal; lemurs; Madagascar; nonrandom dispersal; phylogeny; Primates; seed dispersal; spatial patterns; timing of fruiting; tropical forest.

\section{INTRODUCTION}

Spatial and temporal processes of seed dispersal may play an important role in structuring plant-plant interactions within communities (Hubbell et al. 2001, Terborgh et al. 2002, Russo and Augspurger 2004, Rosindell et al. 2011). These processes set the template for spatial proximities within a community, which, in turn, determine direct and indirect plant-plant interactions (Fort and Richards 1998, Wang and Smith 2002, Russo and Augspurger 2004, Schupp et al. 2010). Importantly, the patterns of spatial proximities resulting from seed dispersal may be nonrandom if seed-dispersing frugivores alter their movements in response to the locations of resources (i.e., fruits) in their environment at a given time (Herrera 2002, Boyer et al. 2006). Since frugivores are the main agents of seed dispersal in many ecosystems (up to $90 \%$, Herrera 2002), understanding these processes may be important for understanding the spatial organization and patterns of recruitment in plant communities (Terborgh et al. 2002). Characterizing how

Manuscript received 9 September 2015; revised 11 March 2016; accepted 15 March 2016. Corresponding Editor: M. Uriarte.

E3E-mail: onja@ricealumni.net animal-mediated dispersal might limit the diversity and frequency of heterospecific interactions between seeds and adult neighbors also has important implications for understanding species coexistence and biodiversity maintenance in diverse plant communities.

Frugivores, which tend to be generalists (Herrera 1982, Bascompte and Jordano 2007, McConkey and Brockelman 2011), spend a large proportion of their time foraging for fruits, and may have a tendency to defecate seeds while feeding (Pratt and Stiles 1983, Clark et al. 2004, Viswanathan et al. 2015). For instance, Clark et al. (2004) reported that birds in Dja Reserve, Cameroon, frequently dispersed seeds under bird-dispersed tree species during their fruiting season. Such behavior could result in nonrandom associations between dispersed seeds and their adult neighbors in terms of fruiting phenology (seasonal timing of fruiting), dispersal mode, and/ or evolutionary history (e.g., if fruiting phenologies or dispersal modes are evolutionarily conserved). While there has been increasing recognition that frugivores often use certain sites, such as fruiting trees, as dispersal foci (a process often referred to as 'contagious dispersal') (Clark et al. 2004, Kwit et al. 2004, Carlo et al. 2007, Blendinger et al. 2011, Watson and Rawsthorne 2013), the resulting pattern of nonrandom associations between 
seeds and adult heterospecifics remains understudied. Given the potential consequences of these nonrandom spatial proximities for recruitment success, understanding how frugivores and timing of fruiting can mediate plant-plant spatial relationships may have important implications for understanding processes that shape plant communities.

One of the main hypothesized advantages of vertebrate seed dispersal is movement away from a parent tree to avoid the associated effects of distance- and densitydependent mortality (Janzen 1970, Connell 1971, Howe and Smallwood 1982). However, once conspecific neighbors are avoided, seed and seedling recruitment success near heterospecific neighbors may vary according to phylogenetic relatedness, which is a commonly used proxy for trait similarities. For example, recent experimental and observational studies suggest that the probabilities of seedling establishment may be predicted by the phylogenetic relatedness between seeds and their adult-neighbors (Vamosi et al. 2009, Burns and Strauss 2011, Liu et al. 2012, Lebrija-Trejos et al. 2014). Plant species that are closely related may share susceptibilities and defenses against natural enemies due to trait conservatism (Webb et al. 2006, Gilbert and Webb 2007, Cavender-Bares et al. 2009), and tend to have similar responses to abiotic factors as well (i.e., habitat filtering, Vamosi et al. 2009, Burns and Strauss 2011). If phylogenetic patterns between dispersed seeds and adult neighbors are at all predictive of seed fate, it is important to understand the role frugivores may play in structuring these interactions. Integrating an evolutionary component into studies of seed dispersal can provide a better understanding of the consequences of frugivore-mediated seed dispersal in a community context.

The spatial patterns of seed dispersal form the initial template for subsequent life-history processes, such as recruitment and survival (Nathan and Muller-Landau 2000, Beckman and Rogers 2013), but it is unclear how nonrandom seed dispersal patterns might translate into spatial proximities of adult plants. By laying the template for plant recruitment, nonrandom seed dispersal could influence neighborhood patterns of adult plant distributions (Levine and Murrell 2003, Rodríguez-Pérez et al. 2012). Alternatively, post-dispersal processes may be so strong that they override initial spatial patterns such that associations found at the seed stage are not predictive of adult distributions and associations (Schupp 1995, Schupp and Fuentes 1995).

In this study, we examined how nonrandom patterns of seed dispersal, resulting from frugivore attraction to fruiting trees (contagious dispersal), affect spatial proximities of seeds and adult trees in a diverse tropical forest. Specifically, we investigated (1) whether fruiting trees are used as dispersal foci by three generalist primate frugivores; (2) how this behavior may structure heterospecific associations between dispersed seeds and adult neighbors in terms of fruiting phenology (seasonal timing of fruiting), dispersal mode, and evolutionary history; and
(3) how nonrandom patterns of neighbor association at the dispersal stage may translate into adult-tree distributions. We applied a combination of direct and indirect dispersal observations, tree community surveys, and a phylogenetic approach to a diverse community of rainforest trees in Madagascar.

\section{Methods}

\section{Study site and system}

This study was carried out in the evergreen montane rainforest of Ranomafana National Park (RNP), located in southeastern Madagascar $\left(47^{\circ} 18^{\prime}-47^{\circ} 37^{\prime} \mathrm{E}\right.$, $21^{\circ} 02^{\prime}-21^{\circ} 25^{\prime} \mathrm{S}$; map in Appendix S1). RNP experiences an average annual rainfall of $2,835 \mathrm{~mm}$, with monthly averages ranging from 10 to $1,200 \mathrm{~mm}$ (Dunham et al. 2011). A peak wet season occurs in January to March (average monthly rainfall of $508 \mathrm{~mm}$ ), and the dry season peaks in June-October (average monthly rainfall: $143 \mathrm{~mm}$ ) (Dunham et al. 2011). Elevation in RNP ranges between $600-1,500 \mathrm{~m}$ (Wright and Andriamihaja 2002). RNP is home to $>330$ known tree and large shrub species (Razafindratsima and Dunham 2015).

We focused our study on the seed dispersal patterns of three arboreal and highly frugivorous lemur species: Eulemur rufifrons (red-fronted brown lemur), Eulemur rubriventer (red-bellied lemur) and Varecia variegata editorum (Southern black and white ruffed-lemur). These species are the largest frugivores in the system (2.0-3.4 kg, Razafindratsima et al. 2013), with body masses at least one kilo heavier than all other frugivores in Ranomafana; and their diets consist of $57-99 \%$ fruit (Wright et al. 2011). Eulemur and Varecia species are generalist frugivores, known to disperse seeds of many plant species in Ranomafana (Dew and Wright 1998, Razafindratsima et al. 2014, Razafindratsima and Dunham 2015) and elsewhere throughout the island (Moses and Semple 2011, Razafindratsima and Martinez 2012, Sato 2012). Our study species are sexually monomorphic in size (Dunham and Rudolf 2009) and travel and forage in small groups (average group size: E. rubriventer: $3.05 \pm$ SD 0.71, E. rufifrons: $6.16 \pm \mathrm{SD}$ 3.48, V. v. editorum: $2.94 \pm \mathrm{SD} 1.23)$.

\section{Tree communities and fruiting phenology}

We used transect surveys to estimate relative abundance and availability of trees within the lemur home ranges. In each of our four study sites where we conducted lemur observations (described below, see map in Appendix S1), we established nine transects of $500 \times 4 \mathrm{~m}$. These transects ran perpendicular to a main trail system and were spaced at least $100 \mathrm{~m}$ apart and overlapped with the home ranges of our study lemur groups. All adult trees in the transects were identified and recorded (Razafindratsima and Dunham 2015). 
Because of logistical constraints, phenological monitoring and spatial mapping of trees were conducted in phenological transects located in only two of our four field sites within RNP. At Valohoaka and Vatoharanana (see map in Appendix S1), we surveyed 10 transects of $100 \mathrm{~m} \times 4 \mathrm{~m}$ (20 transects total). Transects were spaced at least $100 \mathrm{~m}$ apart and ran perpendicular to a major trail. Fruiting occurrences for all canopy trees $(\geq 5 \mathrm{~cm}$ $\mathrm{DBH})$ and understory trees $(\geq 2 \mathrm{~cm} \mathrm{DBH})$ within the transects were monitored every two weeks from July 2012 to June 2014.

\section{Direct observations of seed dispersal by lemurs near fruiting trees}

We quantified seed deposition patterns of the three frugivores through direct observations of lemurdefecation events. We sampled defecations of seeds from eight pre-habituated groups of each of the three lemur species from June 2010 to June 2011, which were distributed across four established field sites within the southern parcel of the park (Mangevo, Talatakely, Valohoaka and Vatoharanana; see map in Appendix S1). We alternated observed groups and species daily (from $0700 \mathrm{~h}$ until about $1700 \mathrm{~h}$ when they were no longer active or we could no longer locate the group), to ensure sampling of comparable data across all groups (Razafindratsima and Dunham 2015). During lemur observations, a team of 2-4 observers attempted to collect all fecal depositions by group members. We identified the species of all seeds within each fecal deposition, and considered conspecific seeds in the same fecal sample as one dispersal event. Also, for each fecal deposition, we identified the species, dispersal mode (lemur or nonlemur) and fruiting status of the nearest adult neighboring tree (i.e., the tree whose canopy was most directly above the defecated seed or whose trunk was nearest to the seed). Tree species were categorized as being dispersed by lemurs or by other means using data from observations of lemur diets, fruit traits, expert knowledge of local botanical and primatological research technicians in RNP, and from the literature (Overdorff 1993, White et al. 1995, Dew and Wright 1998, Rakotomanana et al. 2003, Razafindratsima et al. 2014).

\section{Lemur-mediated seed dispersal into seed traps}

We also sampled lemur-mediated seed dispersal indirectly using seed traps. Each trap was made of durable window screening stapled on a ring of polyvinyl tubing (total trap area $\sim 0.5 \mathrm{~m}^{2}$ ), and hung on trees to a height of $1-1.5 \mathrm{~m}$ to reduce seed removal by granivores (Clark et al. 2004). Traps were placed every $25 \mathrm{~m}$ along the 20 phenology transects described previously. We recorded the identity and dispersal mode (lemur or non-lemur) of all tree species with crowns overhanging each trap (Appendix S2). Every two weeks for 16 months (April 2013-July 2014), we monitored the seed traps, and identified and recorded their contents, including the presence, identity and number of seeds in scat. We also recorded the fruiting status of all trees with crowns overhanging each seed trap.

\section{Analyses: nonrandom seed dispersal in relation to phenology and dispersal mode}

We used a Generalized Linear Model (GLM) in SPSS 22.0 (IBM Inc., Armonk, New York, USA) to examine whether the three frugivores deposited seeds near fruiting, lemur-dispersed trees more frequently than expected by chance (i.e., relative to their availability in the habitat) based on our behavioral observations. Because co-fruiting assemblages vary over time, we used monthly percentage of dispersal events under fruiting trees as our dependent variable, with lemur groups nested within species. To obtain null expectations of random dispersal within the lemur home ranges, we ran multinomial randomization models. The observed seed depositions were randomly paired with neighbor trees that were sampled based on availability of trees in each group's habitat. We assigned an adult tree as fruiting in our model if the species was known to fruit during that month based on observations of tree fruiting status noted during our lemur-dispersal observations and/or in our phenology transects. While this is likely an overestimate of the individuals fruiting in the lemur home ranges, this method made our test of biased-dispersal conservative. Randomizations were run 1000 times in MATLAB R2013b (The MathWorks Inc., Natick, Massachusetts, USA).

We used our seed-trap data to examine whether seed dispersal of species that are known to be lemur-dispersed was biased toward seed traps with one or more fruiting, lemur-dispersed trees overhead, using a Generalized Linear Model with Poisson log-link function. We also performed a Friedman's test to examine whether the seed dispersal of lemur-dispersed trees was higher under the canopy of lemur-dispersed tree species during the fruiting vs. non-fruiting period. These analyses were performed with SPSS 22.0.

\section{Analysis: phylogenetic patterns of seed dispersal}

To conduct our phylogenetic analyses, we used a local phylogenetic tree of all tree species in RNP, reconstructed in Phylomatic v3 using the Angiosperm Phylogeny Group III (AGP3) derived megatree R20120829 (Webb and Donoghue 2005). Branch lengths of the phylogenetic tree were calibrated from estimated molecular and known fossil ages (Wikström et al. 2001) using Phylocom 4.2 (Webb et al. 2008). To estimate the phylogenetic dispersion of tree species categorized as dispersed by lemurs within the community of tree species in RNP, we used Fritz and Purvis' $D$ statistic for discrete traits (Fritz and Purvis 2010). Phylogenetic dispersion (D) was calculated using the package caper, by comparing the observed 
phylogenetic pattern with 1,000 permutations in R 3.0.3 ( $\mathrm{R}$ Core Team 2012), based on random or Brownian motion patterns of evolution. A value of $D>1$ suggests phylogenetic overdispersion, $D=1$ indicates a random trait distribution, $D \sim 0$ indicates trait clustering patterns similar to expected under random genetic drift, and $D<0$ indicates a highly clustered trait (trait conservatism).

We then explored how seed dispersal by lemurs may bias the phylogenetic relationship between dispersed seeds and their adult-tree neighbors. We assessed the phylogenetic relationship between the dispersed seed species and the tree species under which it was deposited. We used a phylogenetic index of relatedness, Net Relatedness Index (NRI) (Webb et al. 2002), to determine if the observed associations were random or biased with respect to phylogeny. $N R I$ estimates a standardized effect size of the mean phylogenetic distance (MPD) between the pairs of seed species and tree species $(s)$ relative to a random distribution $(r)$, using the following equation: $N R I=-1 \times\left[\left(M P D_{s}-M P D_{r}\right) / s d\left(M P D_{r}\right)\right]$. To generate null communities, seed and tree species pairs were randomly drawn without replacement (for a total of 1,000 iterations), from two separate species pools using MATLAB R2013b. The pool for seed species comprised species being dispersed during lemur observations for each lemur group. The tree species pool differed for each group of lemurs and consisted of species found in the $500 \mathrm{~m}$ by $4 \mathrm{~m}$ tree transects (see above) surveyed in each group's territory and included relative abundances. The phylogenetic distances between species pairs were calculated in Phylocom 4.2 (Webb et al. 2008). Similarly, with our seed trap data, we also explored the phylogenetic relationships between dispersed seeds and their adult-tree neighbors (trees with canopies overhead of seed traps). The seed pool used for the null model included a monthly pool of all seeds of lemur-dispersed trees identified as dispersed by a frugivore into the seed trap (observed in scat or with pulp removed; fallen fruits/seeds from overhead trees not included). The tree species pool used for null models for this analysis was based on the transect data as in the previous analysis.

We used one-sample $t$-tests based in group means to determine whether the mean of the estimated values of NRI differed from zero, demonstrating a nonrandom phylogenetic association (Kembel and Hubbell 2006, Razafindratsima et al. 2013). For seed trap data, we also performed serial runs tests (Zar 1999) across samples, using SPSS 22.0, to assess temporal independence of $N R I$ over time, testing the null hypothesis that the distribution of NRI values over time is random (Santorelli et al. 2014).

\section{Analysis: adult-tree distribution}

We examined how patterns of seed dispersal near fruiting trees may be reflected in the distribution of adult trees by examining the tendency of lemur-dispersed trees to have nearest neighbors that are also lemur-dispersed with an overlapping fruiting time. To do this, we used our mapped trees within our phenology transects described previously. For each lemur-dispersed tree (referred to as "focal"), we determined its nearest neighbors in four cardinal directions using the "point-centered quarter" method by Cottam and Curtis (1956). This method consists of dividing the area around the focal tree into four $90^{\circ}$ quarters and measuring the distance from the focal to the closest tree in each quarter. To determine the identity of the nearest neighbor tree species within each quarter angle, we measured the distances between all trees within each plot, and the angles between each pair of trees using the package spatstat in R 3.0.3 (R Core Team 2012). These observed patterns were compared with null expectations, obtained from random shuffling of tree locations using the algorithm rlabel within the $\mathrm{R}$ package spatstat. We performed a Friedman's test to compare the observed and expected mean proportion of neighbor trees that are lemur-dispersed and have overlapping fruiting time with the focal trees.

In two analyses, we used $N R I$ to test the phylogenetic relationships of lemur-dispersed focal trees with their nearest, heterospecific, co-fruiting neighbors relative to random expectations (i.e., randomized neighbors that included all trees in the community). In the first analysis, we calculated $N R I$ when considering only lemur-dispersed neighbors of the focal trees; in the second analysis, we considered all co-fruiting neighbors regardless of dispersal mode.

\section{REsUlts}

\section{Patterns of seed dispersal relative to fruiting}

Overall, the three frugivore species in our study dispersed seeds (through defecation) near fruiting trees upon which they fed, significantly more than expected by chance (Fig. 1; 2-4 times more; GLM, Wald $\left.\chi^{2}=89.97, d f=1, P<0.0001\right)$. This tendency differed significantly among lemur species (Fig. 1; GLM, Wald $\left.\chi^{2}=39.56, d f=23, P=0.017\right)$; however, this result was driven mainly by the greater tendency of $E$. rufifrons and $V$. v. editorum to deposit seeds under conspecific fruiting trees (Appendix S3). Seed dispersal rates under heterospecific, lemur-dispersed trees were similar across frugivore species ( 14-17\%; Appendix S3).

We also examined the rate of dispersal of lemurdispersed seeds into seed traps, determined from seeds found in scat or with pulp removed. Our seed trap data supported our direct dispersal observations of lemurs, such that the rate of frugivore-mediated seed dispersal into a seed trap was significantly higher (2.19 times) if there was a lemur-dispersed tree bearing fruits overhead (Fig. 2A; Wald $\chi^{2}=145.503, d f=1, P<0.0001$ ). The rate of seed dispersal by lemurs under lemur-dispersed tree species was also significantly higher (2.45 times) when the tree was fruiting (Fig. 2B; Friedman's test, $\chi^{2}=5.23$, $d f=1, P=0.02)$; but there was no significant difference 


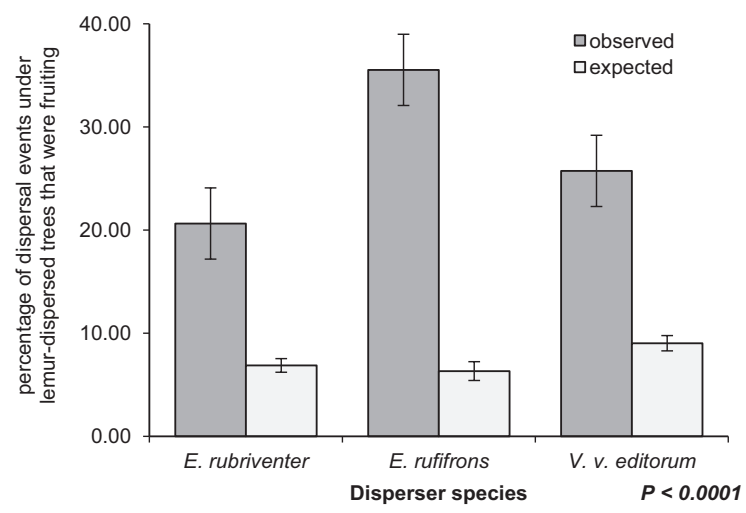

FIG. 1. Patterns of seed-dispersal events near fruiting trees by three frugivore species relative to availability of trees fruiting in their habitats. Error bars represent standard errors across months. $P$-value corresponds to a generalized linear model testing whether the three frugivore species deposit seeds near fruiting, lemur-dispersed trees more frequently than expected by chance (i.e., relative to availability of fruiting trees in the habitat).

in dispersal of seeds under non-lemur-dispersed tree species during fruiting vs. non-fruiting periods (Friedman's test, $\left.\chi^{2}=1.67, d f=1, P=0.20\right)$.

\section{Phylogenetic signal of lemur dispersed plants}

Within our tree community of Ranomafana, we found a phylogenetic signal for tree species dispersed by the three large-bodied lemurs in our study, which differed from the random expectation $(D=0.160, P<0.001)$. In other words, closely related species were more likely to be dispersed by lemurs than expected by chance. The phylogenetic patterns of being dispersed by lemurs did not differ significantly from expectations under a Brownian model of evolution $(P=0.27)$. This indicates that species dispersed by lemurs were clustered on the phylogeny in a pattern no different than expected from a random walk of divergence (drift).

\section{Patterns of phylogenetic relationships between lemur-dispersed seeds and nearest adult neighbor}

We examined the pattern of phylogenetic relatedness between seeds dispersed by lemurs and the adult heterospecific neighbor trees they fell under, by estimating their Net Relatedness Index (NRI), based on our seed trap data. Taking a temporal approach, we found that in nine of the 16 months, the phylogenetic relationships between seeds and adult neighbors were different from random expectations (index values differed from 0 ; Fig. 3). Over time, these patterns fluctuated between clustered (i.e., dispersed seed and its neighbor tree were more closely related than expected by chance) and overdispersed (i.e., dispersed seed and its neighbor tree were more distantly related than expected by chance). However, we found no significant temporal dependence in the monthly distributions of $N R I$ (runs tests, $P=1.00$; Fig. 3).

When all samples across months were pooled, we found that the pattern of relatedness between the dispersed seeds and their adult heterospecific neighbor trees were not different than expected by chance (mean $\left.=-0.035, t_{231}=-0.623, P=0.534\right)$. This was also supported by our direct observations, in which phylogenetic relationships were no different than expected by chance for seeds dispersed by each of the three lemur species individually $\left(\right.$ E. rubriventer: mean $=-0.01, t_{7}=$ $-0.08, P=0.94 ;$ E. rufifrons: mean $=0.01, t_{7}=0.15$,
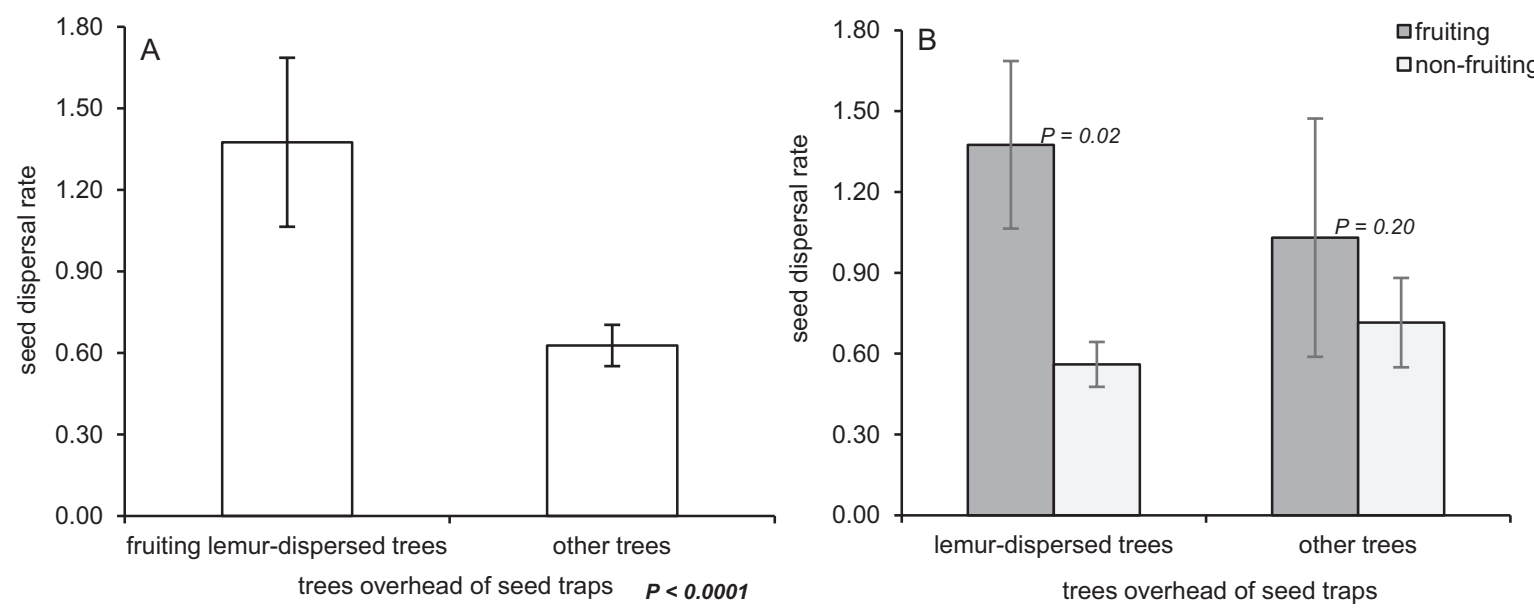

FIG. 2. (A) More seeds are dispersed by lemurs into seed traps under their food trees that are fruiting than under other trees in the environment. (B) More seeds are dispersed by lemurs under lemur-dispersed trees when fruiting than non-fruiting, but this pattern does not hold for trees with other dispersal mechanisms. Error bars represent standard errors. $P$-values corersponds to (A) a generalized linear model examining whether dispersal was biased toward seed traps with a fruiting, lemur-dispersed tree overhead, and (B) Friedman's tests examining whether the dispersal rate of lemur-dispersed seeds was higher under lemur-dispersed tree species during the fruiting vs. non-fruiting period. 


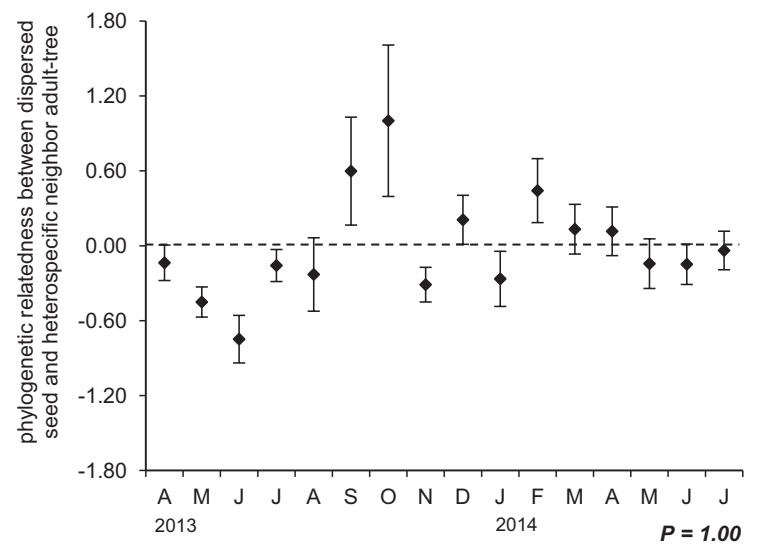

FIG. 3. Phylogenetic relationships (NRI) between dispersed seeds and their nearest adult neighbor trees, using our seed trap data, fluctuate across months (April 2013-July 2014). Values $<0$ indicate that the species pairs are less closely related than expected by chance; $>0$ suggest that they are more closely related than expected by chance, and values near zero indicate a random relatedness. $P$-values correspond to runs test used to assess whether the distribution of $N R I$ values over time is random.

$P=0.89 ; V . v$. editorum: mean $=-0.18, t_{7}=-1.62$, $P=0.15)$. When seed dispersal under conspecific trees was included in the analysis, the phylogenetic relationships of dispersed seeds with nearest adult trees showed a pattern of phylogenetic clustering for those dispersed by $E$. rufifrons $\left(\right.$ mean $\left.=0.50, t_{7}=3.21, P=0.015\right)$ and V. v. editorum $\left(\right.$ mean $\left.=0.30, t_{7}=3.28, P=0.014\right)$, and a pattern no different than random expectation for those dispersed by E. rubriventer (mean $=0.22, t_{7}=1.04$, $P=0.332)$.

\section{Adult-tree distributions}

In our spatial analysis, heterospecific adult, co-fruiting, lemur-dispersed trees were not more likely to be each other's neighbors than expected by chance (observed mean proportion: $0.461 \pm \mathrm{SE} 0.013$, expected mean proportion: $0.457 \pm$ SE 0.013; Friedman's test $\chi^{2}=0.011$, $d f=1, P=0.92$ ). When they were each other's nearest neighbors, these trees showed an overall phylogenetic pattern not different from expected by chance relative to all trees in the community (mean $N R I=-0.045, t_{19}$ $=-1.065, P=0.30)$, However, lemur-dispersed adult trees, were phylogenetically more distant than expected by chance to heterospecific co-fruiting trees in their neighborhoods when heterospecific neighbors of all dispersal modes were included in the analysis (mean NRI $\left.=-0.22, t_{19}=-3.68, P=0.002\right)$.

\section{Discussion}

One way that animal-mediated seed dispersal may impact plants in a community context, is by biasing seed dispersal near certain adult plant species on the landscape. For example, increasing evidence has suggested that it may be common for frugivores to use fruiting trees as seed-dispersal foci (Clark et al. 2004, Blendinger et al. 2011) because of their tendency to defecate or deposit seeds during subsequent feeding bouts. Despite the potential importance of nonrandom associations experienced by seeds for our understanding of plant community structure and diversity (Terborgh et al. 2002), the role frugivores play in structuring seed-adult associations remain understudied, particularly at the community level. We showed that lemur frugivores biased a large proportion of seeds they dispersed near both conspecific and heterospecific fruiting trees upon which they feed; in this way, frugivores may be limiting the frequency and diversity of heterospecific interactions of dispersed seeds. If such dispersal limitation is common in tropical forests where frugivores represent the dominant mode of seed-dispersal, it could promote biodiversity maintenance in these systems by slowing the process of competitive exclusion (Levine and Murrell 2003). Patterns of nonrandom dispersal by frugivores in this system, however, were not predictive of spatial patterns in adult tree neighborhoods, suggesting that post-dispersal processes may be as or more important than seed-dispersal for structuring the fine-scale spatial patterns of adult tree communities.

Lemur-mediated seed dispersal under heterospecific trees also resulted in nonrandom phylogenetic associations between seeds and nearest adult-tree neighbors in nine out of the 16 months of our study. However, these nonrandom phylogenetic associations fluctuated unpredictably over time, ranging from clustered to overdispersed. Interestingly, adult heterospecific neighbors of lemur-dispersed trees that shared dispersers and overlapped in the timing of fruiting were more distantly related than expected by chance given the phylogenetic makeup and abundances of lemur-dispersed trees in the community. This suggests that seeds dispersed by lemurs under fruiting trees that are distantly related may have a better chance at recruitment into the adult stage. Future work to understand these post-dispersal mechanisms may be important for better understanding consequences of seed dispersal and resulting patterns of spatial proximities within adult plant communities.

\section{Seed-dispersal bias under fruiting feeding trees}

The three frugivores in this study dispersed seeds under the canopies of fruiting trees in their diets more than expected from random dispersal. This resulted in nonrandom, positive associations of dispersed seeds with heterospecific co-fruiting adults and with conspecific adults, even when accounting for the relative abundance of all trees in the community. We suspect that this pattern was driven by movement and feeding behavior of the frugivores, and the observation that lemurs tend to defecate while feeding (Overdorff and Strait 1998). 
Regardless of the mechanism, a seed that lands under a heterospecific tree species that is in fruit is likely to experience a high density of heterospecific seeds. For the dispersed seed, this may be better than landing under a parent tree if it is a superior competitor or if seed predators are host-specific and target the seeds of the parent tree (Kwit et al. 2007). However, this situation may also undermine the quality of dispersal because of increased attraction of natural enemies or competitive interactions (Schupp et al. 2002, Russo and Augspurger 2004, Kwit et al. 2007). For seeds landing under conspecific adults, outcomes of seed dispersal may be no greater than landing under a parent tree (Kwit et al. 2007) if distance- or density-dependent mortality is high (e.g., Razafindratsima and Dunham 2015). Seeds of abundant trees may be more likely to experience this fate, as frugivores may visit several trees of the same species within a single day (Carlo et al. 2007). Biased patterns of dispersal towards fruiting trees could also lead to confounding effects for environments experiencing fruit scarcity due to extreme weather events or as a result of habitat disturbance; dispersal limitation and homogenization of the seed bank may occur in these cases (González-Zamora et al. 2014).

The nonrandom patterns of seed dispersal are important on a population and community level because they set the template for post-dispersal processes (Nathan and Muller-Landau 2000, Levine and Murrell 2003, Razafindratsima and Dunham 2015). Our study suggests that post dispersal processes were as strong or stronger than initial seed-dispersal patterns for determining fine-scale patterns of adult distributions (Schupp 1995, Schupp and Fuentes 1995). Neighborhood-scale aggregation of co-fruiting, lemur-dispersed trees at the adult stage were not observed. It is likely that this scale is where direct and indirect interactions between plants and the influence of post-dispersal processes might be the strongest; signatures of seed dispersal might indeed be found on a scale larger than we measured here. However, the lack of consistency between dispersed seed and adult patterns does not negate the importance of our results. In contrast, we suggest such nonrandom dispersal by frugivores is a potential mechanism that might facilitate the maintenance of high levels of tree biodiversity in Madagascar and elsewhere. Seed dispersal that is biased towards certain plant species in a diverse system can result in dispersal limitation and reduced heterospecific interactions (Levine and Murrell 2003). Reduced heterospecific interactions may slow down competitive exclusion (Levine and Murrell 2003), thus acting as an equalizing force that may promote coexistence and help maintain biodiversity (Chesson 2000). If seed dispersal beneath fruiting trees is a common phenomenon in tropical forests where frugivores are the main agents of seed dispersal (Howe and Smallwood 1982, Terborgh et al. 2002), such patterns could help explain how they maintain such high levels of biodiversity.

\section{Phylogenetic patterns of seed dispersal}

The nonrandom patterns of seed dispersal influenced the patterns of phylogenetic relatedness between pairs of dispersed seeds and associated heterospecific neighboring adult-trees, by fluctuating from clustered to overdispersed for the duration of our study period. These fluctuations were unpredictable over time, suggesting that for species with long fruiting times, these phylogenetic relationships may be random overall. However, species with short fruiting times may be more likely to be dispersed near neighbors that are more or less phylogenetically related than expected by chance based on what is co-fruiting at the time. This could have potentially important ecological consequences for these species, given that phylogenetic distance relates to differences in competitive abilities, natural enemies and habitat tolerances, which may influence survival and recruitment of dispersed seeds (Webb et al. 2006, Gilbert and Webb 2007, Cavender-Bares et al. 2009).

Indeed, our adult tree distributions suggest that there may be strong post-dispersal processes limiting recruitment of lemur-dispersed trees near closely related co-fruiting trees. This pattern might arise through heterospecific distance- or density-dependent effects between closely related species (i.e., phylogenetic Janzen-Connell effects) (Cavender-Bares et al. 2009, Liu et al. 2012), acting on the important life-history processes that occur between dispersal and transition to adult plants. Other work has found similar patterns of recruitment. For instance, Liu et al. (2012) found negative effects of phylogenetically similar neighboring adult trees on the survival of Castanopsis fissa seedlings as a result of shared fungal pathogens. However, Lebrija-Trejos et al. (2014) reported improved seedling survival near phylogenetically similar heterospecific neighbors for many species, perhaps a result of habitat filtering. Further research exploring the important role of phylogenetic relatedness in plant recruitment is much needed.

\section{ACKNOWLEDGMENTS}

Research and logistical support was provided by Rice University, MICET and Centre ValBio; and through grants to OHR by Garden Club of America, International Foundation for Science (No. D/4985-1 and D/4985-2), The Rufford Foundation (No. 5748-1), Primate Conservation Inc. (No. 908), The Explorers Club, Idea Wild, and Primate Society of Great Britain. OHR was also supported by Philanthropic Educational Organization, The Leakey Foundation and Schlumberger Foundation. We thank Madagascar National Parks and the Malagasy Ministry of Forest and Water for research permission. We are grateful to M. Andriamalala, M. Rakotondrainibe, N. Beson, J.C. Ramanandraibe, P. Rasabo, J. Solo, G. Razafindrakoto, J. Mamiharilala, A. Pela, Marolahy, Leroa, Velomaro, R. Solohely and P. Rafalinirina for their valuable assistance with fieldwork. Drs. V. Rudolf, H. Rogers, E. Siemann, E. Erhart, C. Masiello, and $\mathrm{K}$. Whitney, and the Dunham research group provided valuable discussion. 


\section{Literature Cited}

Bascompte, J., and P. Jordano. 2007. Plant-animal mutualistic networks: the architecture of biodiversity. Annual Review of Ecology, Evolution, and Systematics 38:567-593.

Beckman, N. G., and H. S. Rogers. 2013. Consequences of seed dispersal for plant recruitment in tropical forests: interactions within the seedscape. Biotropica 45:666-681.

Blendinger, P., J. Blake, and B. Loiselle. 2011. Composition and clumping of seeds deposited by frugivorous birds varies between forest microsites. Oikos 120:463-471.

Boyer, D., G. Ramos-Fernández, O. Miramontes, J. L. Mateos, G. Cocho, H. Larralde, H. Ramos, and F. Rojas. 2006. Scalefree foraging by primates emerges from their interaction with a complex environment. Proceedings of the Royal Society B: Biological Sciences 273:1743-1750.

Burns, J. H., and S. Y. Strauss. 2011. More closely related species are more ecologically similar in an experimental test. Proceedings of the National Academy of Sciences 108:5302-5307.

Carlo, T. A., J. E. Aukema, and J. M. Morales. 2007. Plantfrugivore interactions as spatially explicit networks: integrating frugivore foraging with fruiting plant spatial patterns. Pages 369-390 in A. J. Dennis, R. J. Green, E. W. Schupp, and D. A. Westcott, editors. Seed dispersal: theory and its application in a changing world. CABI International, Oxford, UK.

Cavender-Bares, J., K. H. Kozak, P. V. A. Fine, and S. W. Kembel. 2009. The merging of community ecology and phylogenetic biology. Ecology Letters 12:693-715.

Chesson, P. 2000. Mechanisms of maintenance of species diversity. Annual Review of Ecology and Systematics 31:343-366.

Clark, C. J., J. R. Poulsen, E. F. Connor, and V. T. Parker. 2004. Fruiting trees as dispersal foci in a semi-deciduous tropical forest. Oecologia 139:66-75.

Cottam, G., and J. T. Curtis. 1956. The use of distance measures in phytosociological sampling. Ecology 37:451-460.

Dew, J. L., and P. Wright. 1998. Frugivory and seed dispersal by four species of primates in Madagascar's eastern rain forest. Biotropica 30:425-437.

Dunham, A. E., and V. H. W. Rudolf. 2009. Evolution of sexual size monomorphism: the influence of passive mate guarding. Journal of Evolutionary Biology 22:1376-1386.

Dunham, A. E., E. M. Erhart, and P. C. Wright. 2011. Global climate cycles and cyclones: consequences for rainfall patterns and lemur reproduction in southeastern Madagascar. Global Change Biology 17:219-227.

Fort, K. P., and J. H. Richards. 1998. Does seed dispersal limit initiation of primary succession in desert playas? American Journal of Botany 85:1722-1731.

Fritz, S. A., and A. Purvis. 2010. Selectivity in mammalian extinction risk and threat types: a new measure of phylogenetic signal strength in binary traits. Conservation Biology 24:1042-1051.

Gilbert, G. S., and C. O. Webb. 2007. Phylogenetic signal in plant pathogen-host range. Proceedings of the National Academy of Sciences 104:4979-4983.

González-Zamora, A., V. Arroyo-Rodríguez, F. Escobar, M. Rös, K. Oyama, G. Ibarra-Manríquez, K. E. Stoner, and C. A. Chapman. 2014. Contagious deposition of seeds in spider monkeys' sleeping trees limits effective seed dispersal in fragmented Landscapes. PLoS One 9:e89346.

Herrera, C. M. 1982. Seasonal variation in the quality of fruits and diffuse coevolution between plants and avian dispersers. Ecology 63:773-785.

Herrera, C. M. 2002. Seed dispersal by vertebrates. Pages 185-208 in C. M. Herrera and O. Pellmyr, editors.
Plant-animal interactions: an evolutionary approach. Blackwell Science Ltd, Nashville, Tennessee, USA.

Hubbell, S. P., J. A. Ahumada, R. Condit, and R. B. Foster. 2001. Local neighborhood effects on long-term survival of individual trees in a Neotropical forest. Ecological Research 16:859-875.

Kembel, S. W., and S. P. Hubbell. 2006. The phylogenetic structure of a neotropical forest tree community. Ecology 87:S86-S99.

Kwit, C., D. J. Levey, and C. H. Greenberg. 2004. Contagious seed dispersal beneath heterospecific fruiting trees and its consequences. Oikos 107:303-308.

Kwit, C., D. J. Levey, S. A. Turner, C. J. Clark, and J. R. Poulsen. 2007. Out of one shadow into another: causes and consequences of spatially contagious seed dispersal by frugivores. Pages 427-444 in A. J. Dennis, R. J. Green, E. W. Schupp, and D. Westcott, editors. Seed dispersal: theory and its application in a changing world. CABI, Oxford, UK.

Lebrija-Trejos, E., S. J. Wright, A. Hernández, and P. B. Reich. 2014. Does relatedness matter? Phylogenetic densitydependent survival of seedlings in a tropical forest. Ecology 95:940-951.

Levine, J. M., and D. J. Murrell. 2003. The community-level consequences of seed dispersal patterns. Annual Review of Ecology, Evolution, and Systematics 34:549-574.

Liu, X., M. Liang, R. S. Etienne, Y. Wang, C. Staehelin, and S. Yu. 2012. Experimental evidence for a phylogenetic Janzen-Connell effect in a subtropical forest. Ecology Letters 15:111-118.

McConkey, K. R., and W. Y. Brockelman. 2011. Nonredundancy in the dispersal network of a generalist tropical forest tree. Ecology 92:1492-1502.

Nathan, R., and H. C. Muller-Landau. 2000. Spatial patterns of seed dispersal, their determinants and consequences for recruitment. Trends in Ecology \& Evolution 15:278-285.

Overdorff, D. J. 1993. Similarities, differences, and seasonal patterns in the diets of Eulemur rubriventer and Eulemur fulvus rufus in the Ranomafana National Park, Madagascar. International Journal of Primatology 14:721-753.

Overdorff, D. J., and S. G. Strait. 1998. Seed handling by three prosimian primates in southeastern Madagascar: implications for seed dispersal. American Journal of Primatology 45:69-82.

Pratt, T. K., and E. W. Stiles. 1983. How long fruit-eating birds stay in the plants where they feed: implications for seed dispersal. American Naturalist 122:797-805.

R Core Team. 2012. R: a language and environment for statistical computing. R Foundation for Statistical Computing, Vienna, Austria.

Rakotomanana, H., T. Hino, M. Kanzaki, and H. Morioka. 2003. The role of the Velvet Asity Philepitta castanea in regeneration of understory shrubs in Madagascan rainforest. Ornithological Science 2:49-58.

Razafindratsima, O. H., and A. E. Dunham. 2015. Assessing the impacts of nonrandom seed dispersal by multiple frugivore partners on plant recruitment. Ecology 96:24-30.

Razafindratsima, O. H., S. Mehtani, and A. E. Dunham. 2013. Extinctions, traits and phylogenetic community structure: insights from primate assemblages in Madagascar. Ecography 36:47-56.

Razafindratsima, O. H., T. A. Jones, and A. E. Dunham. 2014. Patterns of movement and seed dispersal by three lemur species. American Journal of Primatology 76:84-96.

Rodríguez-Pérez, J., T. Wiegand, and L. Santamaria. 2012. Frugivore behaviour determines plant distribution: a spatially-explicit analysis of a plant-disperser interaction. Ecography 35:113-123. 
Rosindell, J., S. P. Hubbell, and R. S. Etienne. 2011. The unified neutral theory of biodiversity and biogeography at age ten. Trends in Ecology \& Evolution 26:340-348.

Russo, S. E., and C. K. Augspurger. 2004. Aggregated seed dispersal by spider monkeys limits recruitment to clumped patterns in Virola calophylla. Ecology Letters 7:1058-1067.

Santorelli, S., W. Magnusson, E. Ferreira, E. Caramaschi, J. Zuanon, and S. Amadio. 2014. Phylogenetic community structure: temporal variation in fish assemblage. Ecology and Evolution 4:2146-2153.

Schupp, E. W. 1995. Seed-seedling conflicts, habitat choice, and patterns of plant recruitment. American Journal of Botany 82:399-409.

Schupp, E. W., and M. Fuentes. 1995. Spatial patterns of seed dispersal and the unification of plant population ecology. Ecoscience 2:267-275.

Schupp, E. W., T. Milleron, and S. E. Russo. 2002. Dissemination limitation and the origin and maintenance of species-rich tropical forests. Pages 19-33 in D. J. Levey, W. R. Silva, and M. Galetti, editors. Seed dispersal and frugivory: ecology, evolution, and conservation. CABI, New York, New York, USA.

Schupp, E. W., P. Jordano, and J. M. Gómez. 2010. Seed dispersal effectiveness revisited: a conceptual review. New Phytologist 188:333-353.

Terborgh, J., N. Pitman, M. Silman, H. Schichter, and P. Nunez. 2002. Maintenance of tree diversity in tropical forests. Pages 1-17 in A. J. Dennis, R. J. Green, E. W. Schupp, and D. A. Westcott, editors. Seed dispersal: theory and its application in a changing world. CABI, New York, New York, USA.

Vamosi, S., S. Heard, J. Vamosi, and C. Webb. 2009. Emerging patterns in the comparative analysis of phylogenetic community structure. Molecular Ecology 18:572-592.

Viswanathan, A., R. Naniwadekar, and A. Datta. 2015. Seed dispersal by avian frugivores: non-random heterogeneity at fine scales. Biotropica 47:77-84.
Wang, B. C., and T. B. Smith. 2002. Closing the seed dispersal loop. Trends in Ecology \& Evolution 17:379-385.

Watson, D. M., and J. Rawsthorne. 2013. Mistletoe specialist frugivores: Latterday "Johnny Appleseeds" or self-serving market gardeners? Oecologia 172:925-932.

Webb, C. O., and M. J. Donoghue. 2005. Phylomatic: tree assembly for applied phylogenetics. Molecular Ecology Notes 5:181-183.

Webb, C. O., D. D. Ackerly, M. A. McPeek, and M. J. Donoghue. 2002. Phylogenies and community ecology. Annual Review of Ecology and Systematics 33:475-505.

Webb, C. O., G. S. Gilbert, and M. J. Donoghue. 2006. Phylodiversity-dependent seedling mortality, size structure, and disease in a Bornean rain forest. Ecology 87:123-131.

Webb, C. O., D. D. Ackerly, and S. W. Kembel. 2008. Phylocom: software for the analysis of phylogenetic community structure and trait evolution. Bioinformatics 24:2098-2100.

White, F. J., D. J. Overdorff, E. A. Balko, and P. C. Wright. 1995. Distribution of ruffed lemurs (Varecia variegata) in Ranomafana National Park, Madagascar. Folia Primatologica 64:124-131.

Wikström, N., V. Savolainen, and M. W. Chase. 2001. Evolution of the angiosperms: calibrating the family tree. Proceedings of the Royal Society of London. Series B: Biological Sciences 268:2211-2220.

Wright, P. C., and B. Andriamihaja. 2002. Making a rain forest national park work in Madagascar: Ranomafana National Park and its long-term research commitment. Pages 112-136 in J. Terborgh, C. P. van Schaik, L. Davenport, and R. Madhu, editors. Making parks work: strategies for preserving tropical nature. Island Press, Washington, D.C., USA.

Wright, P. C., S. R. Tecot, E. M. Erhart, A. L. Baden, S. J. King, and C. Grassi. 2011. Frugivory in four sympatric lemurs: implications for the future of Madagascar's forests. American Journal of Primatology 73:585-602.

Zar, J. H. 1999. Biostatistical analysis. Pearson education India.

\section{SUPPORTING INFORMATION}

Additional supporting information may be found in the online version of this article at http://onlinelibrary.wiley.com/ doi/10.1002/ecy.1434/suppinfo 